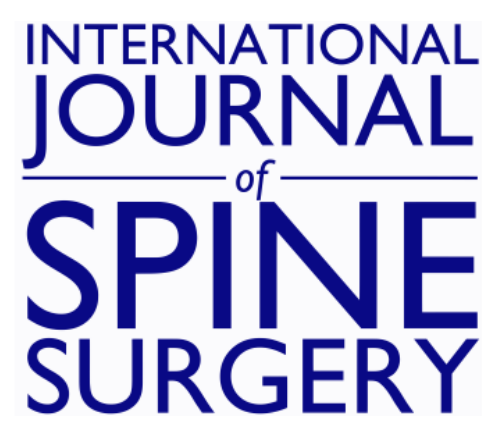

\title{
Facet Arthrodesis with the FFX Device: One-Year Results from a Prospective Multicenter Study
}

\author{
ROBIN SROUR, YASSINE GDOURA, MARIETTE DELAITRE, JIHAD MORTADA, \\ MUSTAPHA ALI BENALI, FABRICE MILLOT, DANIEL HRITCU, ALEXANDRE \\ TIMOFEEV and FRANÇOIS SELLAL
}

Int J Spine Surg 2020, 14 (6) 996-1002

doi: https://doi.org/10.14444/7149

http://ijssurgery.com/content/14/6/996

This information is current as of April 26, 2023.

Email Alerts Receive free email-alerts when new articles cite this article. Sign up at: http://ijssurgery.com/alerts 


\title{
Facet Arthrodesis with the FFX Device: One-Year Results from a Prospective Multicenter Study
}

\author{
ROBIN SROUR, MD, ${ }^{1}$ YASSINE GDOURA, MD,${ }^{1}$ MARIETTE DELAITRE, MD, ${ }^{1}$ JIHAD MORTADA, MD, ${ }^{1}$ \\ MUSTAPHA ALI BENALI, MD ${ }^{2}$ FABRICE MILLOT, MD,${ }^{3}$ DANIEL HRITCU, MD,${ }^{4}$ ALEXANDRE \\ TIMOFEEV, MD, ${ }^{1}$ FRANÇOIS SELLAL, $\mathrm{MD}^{1}$ \\ ${ }^{1}$ Hôpitaux Civils de Colmar, Colmar, France, ${ }^{2}$ Centre Hospitalier Universitaire Dupuytren, Limoges, France, ${ }^{3}$ Hôpital de Saint Avold, Saint Avold, France, \\ ${ }^{4}$ Clinique du Diaconat-Roosevelt, Mulhouse, France
}

\begin{abstract}
Background: Facet osteosynthesis can be performed to treat facet syndrome (FS) and reduce spinal instability following laminectomy in patients with lumbar spinal stenosis (LSS). The present study evaluated clinical and radiological outcomes following facet osteosynthesis with the FFX device.

Methods: Patients with FS or LSS were prospectively enrolled in a single-arm, multicenter study. The device was placed at affected levels with or without concomitant posterior lumbar interbody fusion (PLIF) procedures. The visual analog scale (VAS) for back and leg pain and Oswestry Disability Index (ODI) were evaluated preoperatively and postoperatively. Computed tomography scans to assess fusion and migration were performed 1 year following surgery.

Results: Fifty-three patients (26 men/27 women) with a mean age of $65.0 \pm 9.6$ years (range: $37-83$ years) were enrolled. A total of 205 FFX devices were implanted with 15 patients undergoing concurrent PLIF procedures. There were no intraoperative or postoperative surgical complication reported, and no patient required revision surgery. Mean VAS leg and back pain scores significantly improved from 5.57 to $2.09(P<.001)$ and 5.74 to $3.13(P<.001)$, respectively, between the preoperative and 1 year follow-up assessments. Mean ODI scores also significantly improved from $44.7 \%$ to $24.0 \%(P<.001)$ during the same time period. Facet fusion occurred with $86.3 \%$ of device placements after 12 months. There was $1(0.5 \%)$ asymptomatic device migration. Eight devices $(3.9 \%)$ were considered misplaced.

Conclusions: The use of the FFX device is associated with a significant reduction in both pain and disability following surgery with a high facet joint fusion rate.

Level of Evidence: 4.

Clinical Relevance: This is the first study reporting clinical experience using the FFX device to facilitate facet osteosynthesis. The ability of the device to relieve pain, reduce disability, and enhance lumbar facet fusion with a low rate of device misplacement and migration was demonstrated.
\end{abstract}

New Technology

Keywords: facet arthrodesis, facet joint, facet syndrome, fusion, lumbar canal narrowing, lumbar spinal stenosis, FFX

\section{INTRODUCTION}

Facet joint degeneration is involved in several lumbar spine conditions, whether alone as with facet syndrome (FS), or in combination with other degenerative disorders, as in lumbar spinal stenosis (LSS). ${ }^{1}$ The treatment of degenerative facet conditions ranges from spinal injections or rhizolysis to surgical lumbar decompression with or without laminectomy in cases of patients unresponsive to more conservative treatments. ${ }^{2-4}$ Nonsurgical treatments for FS often only provide temporary pain relief, which typically lasts for a few days to months because the facet joint motion causing the pain is still preserved. ${ }^{2,5}$ For the surgical treatment of LSS, bone removal associated with lumbar decompres- sion and laminectomies can result in the risk of developing spinal instability, potentially leading to spondylolisthesis. ${ }^{6}$ To avoid postdecompression instability, pedicle screw fixation is typically performed during LSS surgeries to accomplish fusion and motion fixation to improve clinical outcomes. ${ }^{6,7}$ Unfortunately, this approach can be associated with (1) a relatively high device misplacement rate, and (2) increased degeneration of the adjacent spinal segments due to the rigidity of pedicle screw fixation systems. $^{8-10}$

The use of facet fusion devices to prevent facet motion represents an interesting alternative for the surgical treatment of patients with FS or LSS. The FFX device (SC Medica, Strasbourg, France) is a 


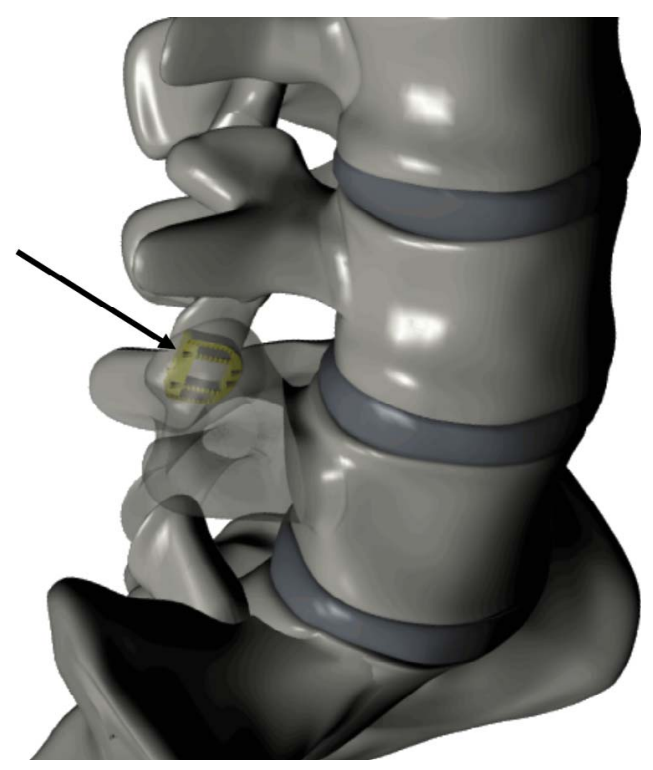

Figure 1. Illustration of the FFX device (arrow) implanted in the L4/L5 facet joint (Courtesy of SC MEDICA).

new implantable facet spacer designed to facilitate facet arthrodesis. The component, which is available in several sizes to ensure proper fit in the facet joint space, is a D-shaped device fabricated in titanium with a serrated surface to facilitate device stabilization. The D is positioned between the facet joints, with its apex oriented anteriorly (Figure 1). The device is surgically placed in the facet joint in combination with bone graft material inside and posteriorly to the $\mathrm{D}$ shape. It is intended to prevent facet motion and relieve pain in patients with FS and to prevent postlaminectomy instability in patients with LSS while avoiding the rigidity associated with conventional pedicle screw osteosynthesis.

We conducted a prospective, multicenter singlearm study to evaluate the use of the FFX device in patients with FS or LSS with the objective of identifying clinical and radiological outcomes associated with single- or multilevel use of the device.

\section{MATERIALS AND METHODS}

This study was a prospective, nonrandomized, observational study conducted at 2 sites between November 2017 and November 2018. A total of 53 consecutive patients with documented LSS or FS received interfacet FFX implants following unsuccessful conservative treatment. The study protocol was approved by a local ethics committee, and patient consent was obtained for all subjects enrolled.
Inclusion criteria for subjects with LSS were a diagnosis of degenerative narrow lumbar canal with the need for decompression confirmed by preoperative magnetic resonance imaging (MRI), computed tomography (CT) scan, or radiculosaccography with radicular claudication while walking and a minimum of 3 months of conservative therapy without symptom improvement. For subjects with FS, inclusion criteria were degenerative lumbar facet joints confirmed by preoperative MRI or CT scan, a diagnostic positive facet joint block for the affected joints, and a minimum of 3 months of conservative therapy without symptom improvement.

Exclusion criteria included radiographically confirmed damage of the vertebral body in the segment of concern in the lumbar spine, isthmic lysis with or without spondylolisthesis, spinal fracture, spondylolisthesis > grade I or other major spinal instability, degenerative lumbar scoliosis $\left(>25^{\circ}\right)$, spondylodiscitis, spinal tumor, osteoporosis, Paget disease, osteomalacia or other metabolic bone disorders, the presumed need for wide resection of facet joints during surgery, more than 5-level involvement, morbid obesity, pregnancy, known allergy to titanium or titanium alloys, and patients who, in the opinion of the investigator, were inappropriate for inclusion in this study.

All subjects were evaluated for pain and disability using the visual analog scale (VAS) for leg and back pain and the Oswestry Disability Index (ODI), preoperatively, at 2 to 3 months, 5 to 6 months, and 12 to 15 months following surgery. CT scans to confirm fusion and assess device migration or misplacement were also performed following a 12month postoperative period. Fusion was defined as any sign of bony fusion inside or posterior to the device when viewing the postoperative CT scan. Migration was defined as any displacement of the device compared with immediate postoperative images. Misplacement was defined as any part of the device being outside the interfacet line in immediate postoperative images. All patient assessments were conducted by independent evaluators who did not perform any of the surgeries.

\section{Operative Technique}

After routine intubation, the patient was placed in the ventral decubitus or genupectoral position. For patients with LSS, an open surgery was performed, and a middle sagittal incision was made with respect to the lumbar canal narrowness. For 
Table. Demographic and surgical data.

\begin{tabular}{|c|c|}
\hline Parameter & Value \\
\hline \multicolumn{2}{|l|}{ Sex, n $(\%)$} \\
\hline Men & $26(49.1)$ \\
\hline Women & $27(50.9)$ \\
\hline Age, mean (range), y & $65.5(37-83)$ \\
\hline \multicolumn{2}{|l|}{ Number of surgical levels, n (\%) } \\
\hline 1 level & $24(47.1)$ \\
\hline 2 levels & $14(27.5)$ \\
\hline 3 levels & $8(15.7)$ \\
\hline 4 levels & $6(11.3)$ \\
\hline 5 levels & $1(1.9)$ \\
\hline \multicolumn{2}{|l|}{ Mean number of FFX devices per level } \\
\hline All patients & 1.95 \\
\hline 1 level & 2.00 \\
\hline 2 levels & 1.82 \\
\hline 3 levels & 2.00 \\
\hline 4 levels & 2.00 \\
\hline 5 levels & 2.00 \\
\hline \multicolumn{2}{|c|}{$\begin{array}{l}\text { FFX implant levels (number of patients with } \\
\text { concurrent PLIF procedures) }\end{array}$} \\
\hline L3-L4 & $8(5)$ \\
\hline L4-L5 & $11(4)$ \\
\hline L5-S1 & $5(2)$ \\
\hline L1-L2; L2-L3 & $1(0)$ \\
\hline L2-L3; L3-L4 & $4(1)$ \\
\hline L3-L4; L4-L5 & $4(1)$ \\
\hline L3-L4; L5-S1 & $2(2)$ \\
\hline L4-L5; L5-S1 & $3(0)$ \\
\hline L1-L2; L2-L3; L3-L4 & $6(0)$ \\
\hline L2-L3; L3-L4; L4-L5 & $2(0)$ \\
\hline L1-L2; L2-L3; L3-L4; L4-L5 & $4(0)$ \\
\hline L2-L3; L3-L4; L4-L5; L5-S1 & $2(0)$ \\
\hline L1-L2; L2-L3; L3-L4; L4-L5; L5-S1 & $1(0)$ \\
\hline
\end{tabular}

Abbreviation: PLIF, posterior lumbar interbody fusion.

patients with FS, 2 side incisions were performed to allow access to the facet joints via a minimally invasive transmuscular approach. Paravertebral muscle dissection was performed in front of the facet joints on both the left and right sides. Exposure of the articular line spacing for each facet was performed with a facet chisel (and/or periosteal elevator) followed by a reviving of the facet joints with a rasp to promote fusion. Two implants were used per level. After connecting the implant onto the facet holder, bone graft material was inserted into the empty space of the device. While attached to the facet holders and at the entry of the articular lines, the devices were inserted into the facet joints simultaneously on the right and left sides. The devices were then pushed into place using a supplied impactor and positioned appropriately.

For patients with LSS, laminectomy and canal recalibration were performed after the above. Bone graft material was also added posterior to the inserted implants. If a patient underwent a discectomy, a posterior lumbar interbody fusion (PLIF) procedure was performed using standard operative technique placing 2 interbody cages bilaterally.
Surgical wounds were closed and sutured per standard routine following completion of the procedure.

All surgeons involved in the study were required to complete a minimum of 5 procedures with the FFX device prior to participating in the study.

\section{Statistical Methods}

Statistical significance of the observed changes in VAS back and leg pain and ODI scores were assessed using paired two-sided $t$ tests. All analyses were performed using $\mathrm{R}$ v3.6.2.

\section{RESULTS}

A total of 205 FFX devices were implanted in 53 patients at 2 centers. Patients enrolled in the study included 26 men and 27 women with a mean patient age of $65.0 \pm 9.6$ years (range: $37-83$ years). Fifty of the 53 patients $(94.3 \%)$ had been diagnosed with LSS and $3(5.7 \%)$ with FS. The Table summarizes the number of levels operated on, FFX devices implanted, mean number of implants per level, and concurrent PLIF procedures performed in the patient population. Twenty-four patients had single-level FFX procedures, 14 had 2-level, 8 had 3level, 6 patients had 4-level, and 1 patient had a 5level procedure. There was a mean of 1.95 FFX devices implanted per level with 49 patients $(92.5 \%)$ having devices placed bilaterally at all operative levels. No intraoperative or postoperative surgical complications occurred, and revision surgery was not required for any of the patients enrolled the study.

Fifteen of the 53 patients $(28.3 \%)$ had concurrent PLIF procedures in conjunction with FFX device placement. Patients receiving PLIFs were slightly older $(68.1 \pm 8.3$ years $)$ than patients not having a concurrent PLIF procedure (64.1 \pm 9.9 years). The PLIF group also had a higher percentage of men $(53.3 \%)$ compared with the non-PLIF group (47.4\%). The PLIF group consisted of 11 patients undergoing single-level procedures $(73.3 \%)$ and 4 patients having 2-level procedures $(26.7 \%)$. This contrasted with 13 patients in the non-PLIF group having single-level procedures $(34.2 \%)$ and 10 patients with 2-level procedures $(29.4 \%)$. No patients in the PLIF group had more than 2 levels operated on compared with 11 patients in the nonPLIF group. 

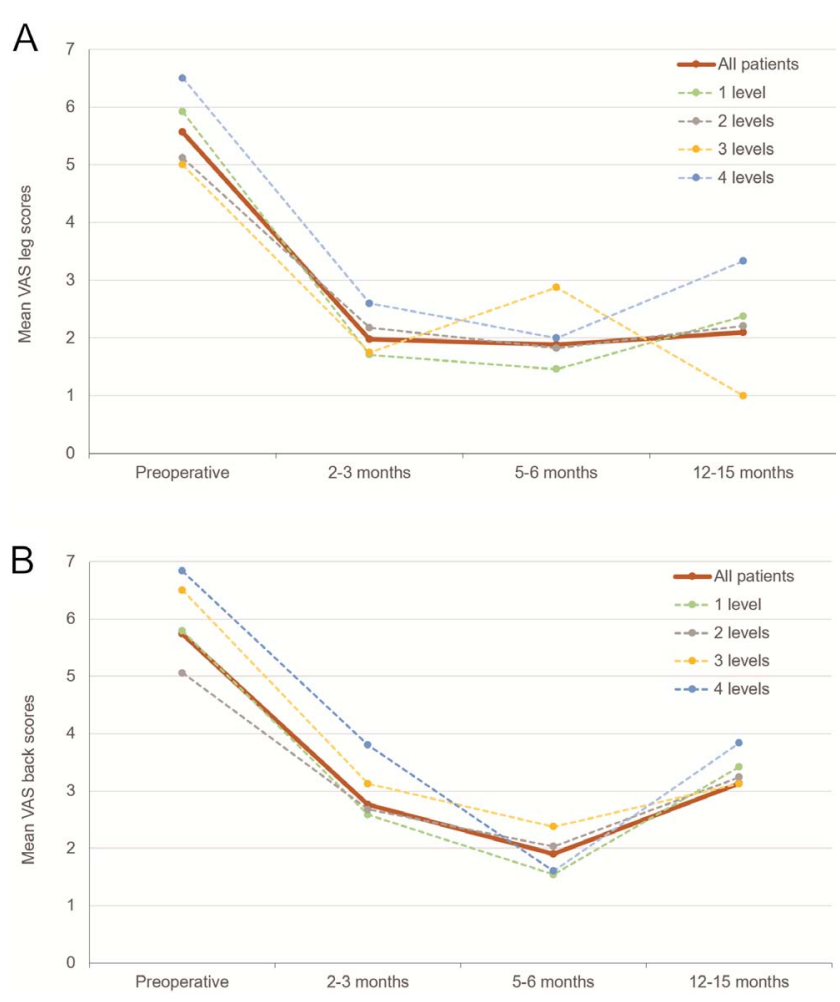

Figure 2. Mean visual analog scale (VAS) scores for (A) leg pain and (B) back pain; by level. VAS scores significantly improved $(P<.05)$ at all assessment intervals versus preoperative VAS scores for both leg and back pain for patients receiving FFX implants for 1 to 4 levels. Excludes 2 patients for whom 2- to 3 month and 5- to 6-month data were not available.

Mean VAS scores for leg pain showed a statistically significant improvement from 5.57 preoperatively to $2.09(P<.001)$ after 12 to 15 months (mean 13.1 months) for the entire patient population. Patients in the non-PLIF group also had a significant improvement in VAS leg pain scores (5.11 preoperatively to 2.30 after $12-15$ months, $P=.002)$. There was a trend toward improvement in the PLIF group (6.60-1.73, $P=$ .056). Mean VAS scores for back pain for the entire study population significantly improved from 5.74 to $3.13(P<.001)$ during the observation period. Similar to the above, patients in the non-PLIF group also had a significant improvement in VAS back pain scores (5.78 preoperatively to $3.24, P=$ .003). Although there was a reduction in VAS back pain scores in the PLIF group (5.33-2.60), this did not reach statistical significance $(P=.355)$ likely because of the limited number of patients in this group. Forty-one $(77.4 \%)$ and $39(73.6 \%)$ of the patients enrolled in the study showed improvements in their leg and back VAS scores, respectively, after 12 months.

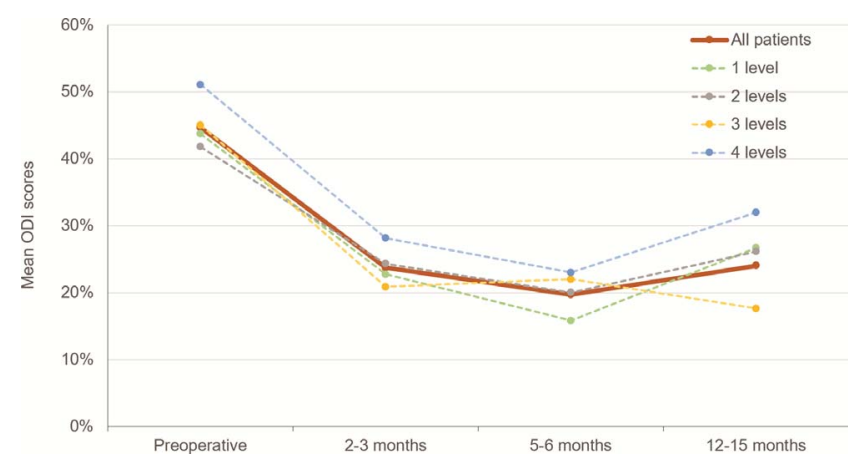

Figure 3. Mean Oswestry Disability Index (ODI) scores, by level. ODI scores significantly improved $(P<.05)$ at all assessment intervals versus preoperative ODI scores for patients receiving FFX implants from 1 to 4 levels. Excludes 2 patients for whom 2- to 3-month and 5- to 6-month data were not available.

Mean ODI scores also showed a statistically significant improvement decreasing from $44.7 \%$ preoperatively to $24.0 \%(P<.001)$ postoperatively at the end of the study observation period. Mean ODI scores improved significantly for both the nonPLIF and PLIF groups $(45.4 \%-25.8 \%, P=.001$; and $42.4 \%-18.3 \%, P=.039)$ across the same time period. Forty-six patients $(86.8 \%)$ had improved ODI scores after 12 months.

VAS and ODI scores were not available for 2 patients $(3.8 \%)$ at the 2- to 3-month and 5- to 6month assessment intervals. Mean VAS and ODI scores for the remaining patients with data available for all 3 of the postoperative follow-up periods showed significant improvements at both 2 to 3 months and at 5 to 6 months $(P<.001)$ compared with the preoperative scores. The above findings were observed across all subjects independent of the number of levels operated on (Figures 2A, 2B, and 3 ). There were no statistically significant differences in leg or back VAS scores or ODI scores between the 2- to 3-month and the post 12-month follow-up period when analyzed for the entire patient population or for either the PLIF or non-PLIF groups when analyzed separately. This suggests an early and maintained achievement of improved outcomes. Although VAS and ODI scores increased between the 6-month and post 12-month follow-up period in some patient subgroups, these increases were not statistically significant for leg VAS and ODI scores $(P<.008$ for back VAS $)$, and all pain and disability scores after 12 months remained significantly lower than preoperative scores for all patients regardless of how many levels were involved or if the patient received a PLIF or not.

Visual inspection of the 12-month CT scans showed facet fusion was associated with $86.3 \%$ of 


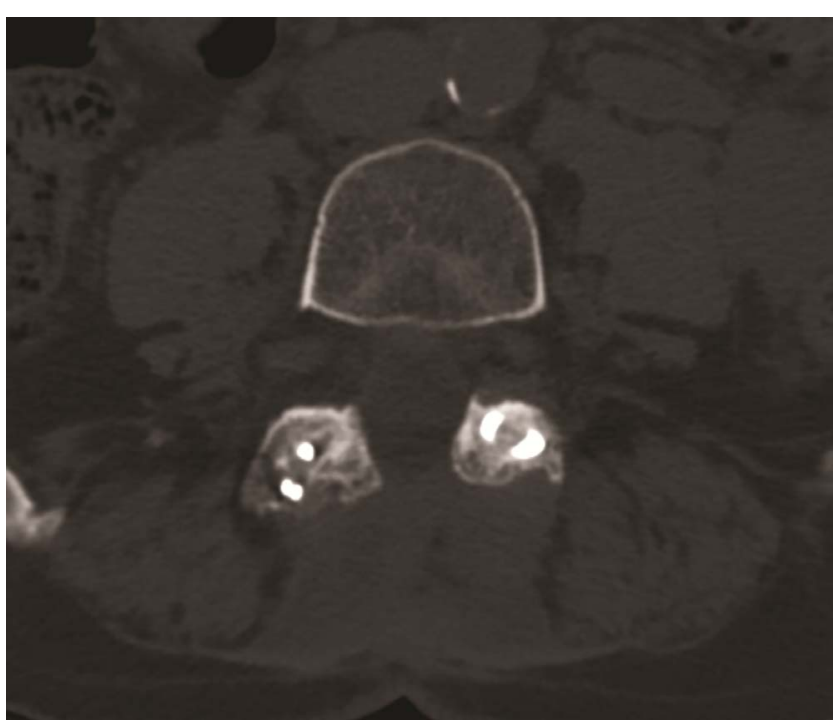

Figure 4. Twelve-month postoperative computed tomography (CT) scan showing facet fusion with the FFX device.

device placements (Figure 4). The facet fusion rate was $88.7 \%$ in the non-PLIF group and $75.7 \%$ in the PLIF group. Although one patient had a single asymptomatic device migration $(0.5 \%)$, this patient's VAS and ODI scores all improved after 12 months. Eight devices $(3.9 \%)$ were considered misplaced by the independent evaluator (Figure 5).

\section{DISCUSSION}

The present study assessed the use and clinical outcomes of the FFX device in patients with LSS or FS. The FFX device is designed to help achieve facet fusion and spinal stability. This new surgical approach demonstrated the ability to achieve a reduction in both pain and disability with a high percentage of patients achieving facet fusion. Overall, $49(92.5 \%)$ of the patients in the present series showed improvements in either their VAS or ODI scores after 12 months.

The procedure associated with the placement of the FFX device was straightforward and no intraor postoperative surgical complications were noted. All investigators were required to have performed a minimum of 5 procedures with the device prior to enrolling patients into the study to ensure adequate training and experience with implanting the device. The technique for placing the device was found to be simple and associated with a rapid learning curve. Eight devices (3.9\% of all devices placed) were classified as being misplaced by an independent evaluator as a result of a small portion of the device

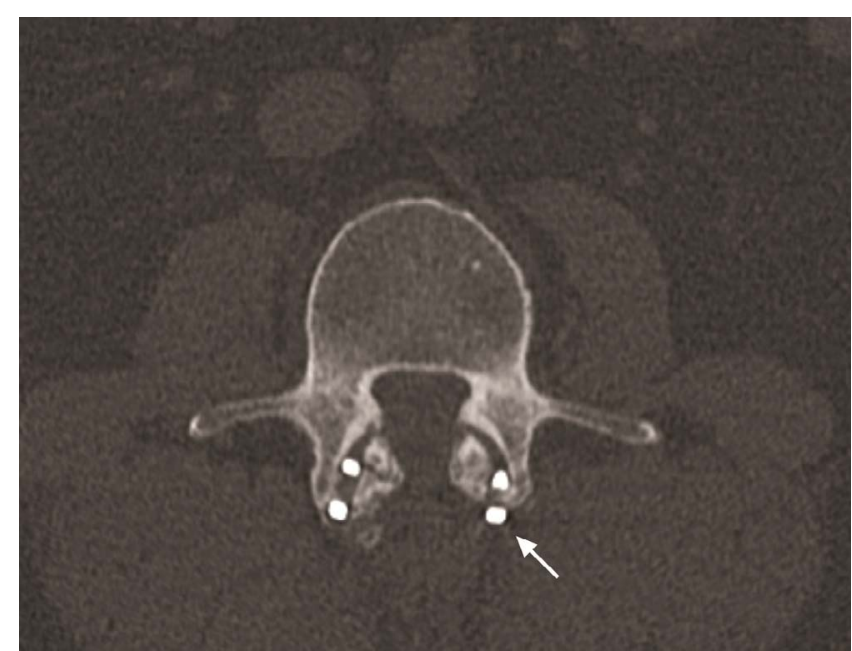

Figure 5. Computed tomography (CT) scan showing example of FFX device misplacement (arrow).

being outside the interfacet line (Figure 4). This low misplacement rate compares favorably with the $12.7 \%$ inaccuracy rate reported in a meta-analysis of nonnavigated pedicle screw placements $(7.9 \%$ with navigation) performed during lumbar procedures. ${ }^{8}$ Additionally, FFX device misplacement did not result in the need for reoperation.

The FFX device was placed bilaterally at all levels in 49 of the 53 patients $(92.5 \%)$ enrolled in the study regardless of the presence of unilateral or bilateral symptoms. Unilateral implantation of the device was performed in patients where 1 facet joint was fused prior to the surgery. All 4 patients with unilateral implants showed improvements in both VAS and ODI scores following surgery. PLIF procedures were performed in conjunction with FFX device implant placement for $15(28.3 \%)$ of the patients enrolled in the study who had a lumbar discectomy performed as a part of the operative procedure.

Among the patients where improvement in back VAS was not observed after 12 months, fusion was seen in all of the devices implanted for 7 of 14 patients $(50 \%)$ with 7 patients $(50 \%)$ not having fusion for all devices. Similar findings were observed for patients who did not have improvements in leg VAS (5 of 12 had fusion for all devices and 7 of 12 did not) or ODI ( 3 of 7 had fusion for all devices and 4 of 7 did not). These findings suggest that there was no relationship between VAS and ODI scores and fusion rate. The lack of a link between nonfusion and the absence of improvement after 12 months compared with preoperative scores may 
be related to the more stringent criteria used to define fusion compared with other studies. ${ }^{13}$ For the present study, fusion was defined as signs of "bony" fusion, whereas Goel et $\mathrm{al}^{13}$ defined fusion as the absence of motion and alterations in the interlaminar and intervertebral body distances on flexionextension radiographs obtained at a follow-up. Because the FFX device blocks facet motion, the fusion rate reported in the present study would likely have been increased if this less constraining criteria had been utilized.

A single patient experienced asymptomatic device migration that did not require reoperation. No patients enrolled in the study required revision surgery. There was no identified compression of adjacent foramina or accelerated foraminal stenosis resulting from facet distraction produced by the device. There were also no accelerated degenerative changes identified at the post 12-month follow-up.

The FFX device may provide a potential improved alternative to other approaches utilized for the treatment of FS. Current approaches to the treatment of primary FS or secondary to lumbar arthroplasty ${ }^{11}$ include steroid injections, radiofrequency ablation (rhizolysis), cryoneurolysis, and chemical neurolysis with phenol or alcohol. ${ }^{12}$ Although effective, these approaches often only provide temporary relief of pain in many patients. For patients with FS, facet arthrodesis can be performed in a minimally invasive manner with the FFX device and may represent an effective long-term treatment, given the ability of the device to prevent facet joint motion potentially causing pain.

The FFX device provides several potential advantages to the use of pedicle screw fixation systems in patients with LSS receiving laminectomies where postsurgical spinal stabilization is desired. The less rigid fixation and reduced load projects with the FFX device compared with pedicle screws would theoretically result in less adjacent segment degeneration and reduced need for subsequent surgical procedures. Additionally, the placement of the FFX device does not inhibit the ability to perform a fusion procedure with pedicle screw placement in the future, if desired. Although there was a low misplacement rate with the FFX device in the present study, this compared favorably to the reported misplacement rate for pedicle screws. Additionally, even though there was only 1 case of device migration in this study, FFX device migra- tion would generally result in the device ending up in the muscle, a significant advantage versus the risks associated with pedicle screw misplacement in the canal and the potential need for reoperation to avoid permanent injuries or disabilities.

Because the FFX device can be placed under direct visualization, the procedure avoids the use of fluoroscopy and associated radiation risks that are required for pedicle screw placement. Additionally, the reduced surgical exposure required for the procedure and simpler placement technique compared with the pedicle screw fixation systems translates to the potential for a reduction in operative time and blood loss for patients. A recent nonrandomized, retrospective study compared mean operative time and estimated blood loss between patients undergoing posterior lumbar fusion surgery for LSS with either pedicle screw fixation or the FFX device. ${ }^{14}$ The author reported that pedicle screw fixation was associated with significantly longer mean operative time compared with placement of the FFX device (152.5 \pm 39.4 versus $99.4 \pm 44.0$ minutes; $P<.001)$. Mean operative blood loss was also significantly greater for pedicle screw versus FFX procedures (446.5 \pm 272.0 versus $251.0 \pm 315.9 \mathrm{~mL} ; P<.001)$. These differences were independent of the number of levels operated on.

There are several potential limitations associated with the present study. The use of a nonrandomized study design and the resultant lack of a control group prevents the ability to directly compare the results obtained to those achieved with standard approaches utilized for patients with LSS and FS. The 12- to 15-month follow-up period also limits the ability to project outcomes associated with the device beyond this time period. The limited number of patients in this series diagnosed with FS limits the ability to generalize our results to this patient population.

\section{CONCLUSIONS}

The present study represents the first clinical assessment of the FFX device in patients with LSS and FS. Clinical outcomes showed a reduction in both pain and disability following surgery with a high fusion rate. Additional studies are needed to assess the long-term results of facet fixation and to directly compare this approach with other procedures and fixation constructs. 


\section{ACKNOWLEDGMENTS}

The authors thank Samvida Venkatesh for performing the statistical analysis for this study. The present study was approved by the Institutional Review Board at Hôpitaux Civils de Colmar.

\section{REFERENCES}

1. van Kleef M, Vanelderen P, Cohen SP, Lataster A, Van Zundert J, Mekhail N. Pain originating from the lumbar facet joints. Pain Pract. 2010;10(5):459-469. doi:10.1111/j.1533-2500. 2010.00393.x

2. Chou R, Hashimoto R, Friedly J, et al. Epidural corticosteroid injections for radiculopathy and spinal stenosis: a systematic review and meta-analysis. Ann Intern Med. 2015;163(5):373-381. doi:10.7326/M15-0934

3. Manchikanti L, Abdi S, Atluri S, et al. An update of comprehensive evidence-based guidelines for interventional techniques in chronic spinal pain. Part II: guidance and recommendations. Pain Physician. 2013;16(2 suppl):S49-283.

4. Zaina F, Tomkins-Lane C, Carragee E, Negrini S. Surgical versus nonsurgical treatment for lumbar spinal stenosis. Spine. 2016;41(14):E857-E868. doi:10.1097/BRS.0000 000000001635

5. Tomé-Bermejo F, Barriga-Martín A, Martín JL. Identifying patients with chronic low back pain likely to benefit from lumbar facet radiofrequency denervation: a prospective study. $J$ Spinal Disord Tech. 2011;24(2):69-75. doi:10.1097/BSD. 0b013e3181dc9969

6. Guha D, Heary RF, Shamji MF. Iatrogenic spondylolisthesis following laminectomy for degenerative lumbar stenosis: systematic review and current concepts. Neurosurg Focus. 2015;39(4):E9. doi:10.3171/2015.7.FOCUS15259

7. Fischgrund JS, Mackay M, Herkowitz HN, Brower R, Montgomery DM, Kurz LT. 1997 Volvo Award winner in clinical studies: degenerative lumbar spondylolisthesis with spinal stenosis: a prospective, randomized study comparing decompressive laminectomy and arthrodesis with and without spinal instrumentation. Spine. 1997;22(24):2807-2812. doi:10. 1097/00007632-199712150-00003

8. Kosmopoulos V, Schizas C. Pedicle screw placement accuracy: a meta-analysis. Spine. 2007;32(3):E111-E120. doi:10. 1097/01.brs.00002540

9. Aota Y, Kumano K, Hirabayashi S. Postfusion instability at the adjacent segments after rigid pedicle screw fixation for degenerative lumbar spinal disorders. $J$ Spinal Disord 1995;8(6):464-473.

10. Park P, Garton HJ, Gala VC, Hoff JT, McGillicuddy JE. Adjacent segment disease after lumbar or lumbosacral fusion: review of the literature. Spine. 2004;29(17):1938-1944. doi:10. 1097/01.brs.0000137069.88904.03

11. Punt IM, Visser VM, van Rhijn LW, et al. Complications and reoperations of the SB Charite lumbar disc prothesis: experience in 75 patients. Eur Spine J. 2008;17(1):36-43. doi:10. 1007/s00586-007-0506-8

12. Perolat R, Kastler A, Nicot B, et al. Facet joint syndrome: from diagnosis to interventional management. Insights Imaging. 2018;9(5):773-789. doi:10.1007/s13244-0180638-x

13. Goel A, Shah A, Jadhav M, Nama S. Distraction of facets with intraarticular spacers as treatment for lumbar canal stenosis: report on a preliminary experience with 21 cases. $J$ Neurosurg Spine. 2013;19(6):672-677. doi:10.3171/2011.8. SPINE11249

14. Srour R. Comparison of operative time and blood loss with the $\mathrm{FFX}^{\oplus}$ device versus pedicle screw fixation during surgery for lumbar spinal stenosis: a retrospective cohort study. medRxiv. 2020;doi:10.1101/2020.05.09.20096651

Disclosures and COI: Neither of the authors involved in the evaluation of the patients report having conflicts of interest related to this work. RS reports having a relative that is employed by $\mathrm{SC}$ Medica and being a designer in the patents of the technologies. The remaining authors report no conflict of interest.

Corresponding Author: Robin Srour, MD, Hôpitaux Civils de Colmar, 39 Avenue de la Liberté, 68024 Colmar, France. Phone: +(33) 389 1245 76; Email: robin.srour@ch-colmar.fr.

Published 22 January 2021

This manuscript is generously published free of charge by ISASS, the International Society for the Advancement of Spine Surgery. Copyright (C) 2020 ISASS. To see more or order reprints or permissions, see http://ijssurgery.com. 